\title{
Initial experience with implantation of novel dual layer flow-diverter device FRED
}

\author{
Wojciech Poncyljusz' ${ }^{1}$ Leszek Sagan², Krzysztof Safranow³, Monika Rać3 \\ 1Department of Interventional Radiology, Pomeranian Medical University, Neurointerventional Cath Lab MSW Hospital, Szczecin, Poland \\ 2Department of Neurosurgery and Paediatric Neurosurgery, Pomeranian Medical University, Szczecin, Poland \\ ${ }^{3}$ Department of Biochemistry and Medical Chemistry, Pomeranian Medical University, Szczecin, Poland
}

Videosurgery Miniinv 2013; 8 (3): 258-264

DOI: 10.5114/wiitm.2011.35794

\begin{abstract}
Flow-diverting stents can help treat complex and wide-necked cerebral aneurysms. The aim of the study was to evaluate initial experiences related to the safety and effectiveness of eight aneurysms treated with a new dual layer coverage designed flow-diverter device. In 2012 Fred flow-diverter devices were used to treat 8 unruptured wide neck (dome-neck ratio $\leq 1.5$ ) and sidewall aneurysms in 6 patients. All aneurysms were located in the anterior circulation on the internal carotid artery (ICA). In 4 larger aneurysms (>10 mm) one 3D coil in association with Fred was used to reduce potential incidence of postoperative subarachnoid haemorrhage (SAH). Dual antiplatelet therapy was administered before the procedure and continued for 3 months after it. Clinical parameters, aneurysm features and 3-month follow-up angiograms are presented. All 6 patients with 8 aneurysms were successfully stented with the Fred flowdiverter device and were discharged in generally good condition on dual-antiplatelet therapy. No complications were related to the procedure. In 5 cases digital subtraction angiography (DSA) control examination was performed after 3 months, showing complete occlusion of the aneurysms with patency of the parent artery. In 1 case thrombosis of the Fred occurred but without any clinical consequences because of cross-flow from the other side. Use of the Fred flow-diverter device was efficacious in all 8 treated cerebral aneurysms. The system seems to be promising as a flow diverter with certain characteristics, which allow for easy delivery and implantation. Further clinical evaluation with a larger group of patients is needed.
\end{abstract}

Key words: intracranial aneurysms, embolization, flow diverters, Fred device.

\section{Introduction}

Recently flow diverters became very important tools in the treatment of intracranial aneurysms, with acceptable rates of aneurysm occlusion, morbidity, and mortality [1-15]. These devices continue to strengthen their position through reconstruction of the vessel walls in the treatment of complex brain aneurysms [1-15]. A large number of them were implanted in patients; however, occlusion, recanalization and compli- cation rates have not been fully understood in detail so far [16]. New devices with different structure appear on the market; therefore understanding of safety and efficacy profiles associated with new flow diverters in treatment of intracranial aneurysms should be taken into special consideration.

We report the first case series of patients treated with a new dual working layer flow-diverter system that is a self-expanding, single wire braid, compliant 
closed-cell design, more pliable compared to the currently used generation of flow diverters (Photo 1).

\section{Case report}

All 6 patients were recruited from the neurosurgery department of the regional medical university. They signed consent to participate in this new device series study which was approved by the local ethic committee. The Fred device has a European Union certificate. All procedures were discussed at the meeting between the neurointerventional radiologist and neurosurgeons.

The entry criteria included unruptured widenecked internal carotid aneurysms. Exclusion criteria included presence of haemodynamically significant atherosclerotic lesions in the carotid artery at the same side, intolerance to heparin or resistance to anti-platelet therapy, coagulopathies, and abnormal platelet count. Initial evaluation of the aneurysm was based on computed tomography (CT) angiography and assessed on reconstructions. Clinical examination was performed before the procedure, patients' discharge from hospital and after 3-month follow-up. digital subtraction angiography (DSA) was obtained before, after the procedure and then after 3-month follow-up which was subsequently analyzed by a neurointerventional radiologist and neurosurgeon. Xpert-CT was additionally performed in all cases after Fred implantation for opening flow diverter evaluation. All the demographic and clinical characteristics of our patients are presented in Table I. In 5 cases patients had a history of another previously treated ruptured aneurysm. All patients reported headache, one had limited field of view and ptosis, and one reported facial numbness. No other neurological deficits were observed.

All procedures were performed in 2012, under general anaesthesia by one experienced neurointerventional radiologist. After the puncture of the common femoral artery and insertion of a $6 \mathrm{~F}$ sheath, a bolus of $5000 \mathrm{IU}$ of heparin was administered. Then, as an access-guiding catheter Chaperon $6 \mathrm{~F}$ (Microventiona-Terumo) over guide-wire was positioned in the internal carotid artery (ICA) and selective DSA was performed. Crossings of the aneurysm neck were performed with a delivery microcatheter (Headway 0.027) and microguidewire (Traxcess 0.014) under roadmap guidance. In 4 cases with big aneurysms an Echelon 10 (EV3) microcatheter was placed in the

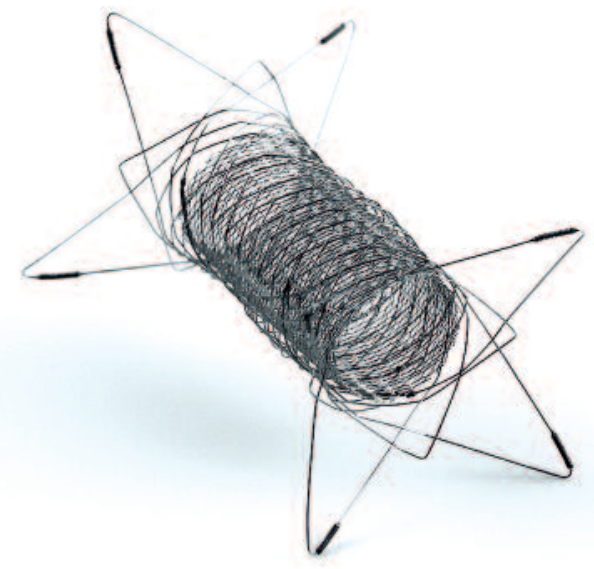

Photo 1. Fred flow-diverter system demonstrating dual working mid-section layer marked with radio-opaque helix to provide fluoroscopic visibility, distal and proximal markers evenly dispersed on its single layer ends

aneurysm sack to deliver a 3D coil before stent deployment. In 1 case with two ICA aneurysms where one was previously treated with coils and on 6month follow-up flow inside coils was present. Fred was deployed on two aneurysms in this case. The central portion of the Fred was placed just at the site of the aneurysm neck. In all cases passing and deployment were very easy using proximal and distal markers of the device, which demonstrated full deployment of it. Positioning of the working inner stent layer based on helical strands could be possible on very good quality fluoroscopy because of the border poor visibility between the single and double (working) layer of the flow diverter. Then 3D DSA was performed in all cases at the end of the procedure to demonstrate haemodynamic changes and the final result with flow inside the flow diverter. Xpert-CT was used to show flow diverter structure and full opening (Photos $2 \mathrm{~A}$ and B). Acetylsalicylic acid in a dose of $75 \mathrm{mg} /$ day and clopidogrel in a dose of $75 \mathrm{mg} /$ day, respectively, were administered 5 days before the planned procedure and continued for 3 months after it. Before their discharge, all patients underwent neurological examinations using the modified Rankin score performed by a neurosurgeon.

Initial technical success of the treatment was defined as proper implantation of the device across the neck of the aneurysm without any technical problems. The clinical success before discharge and 


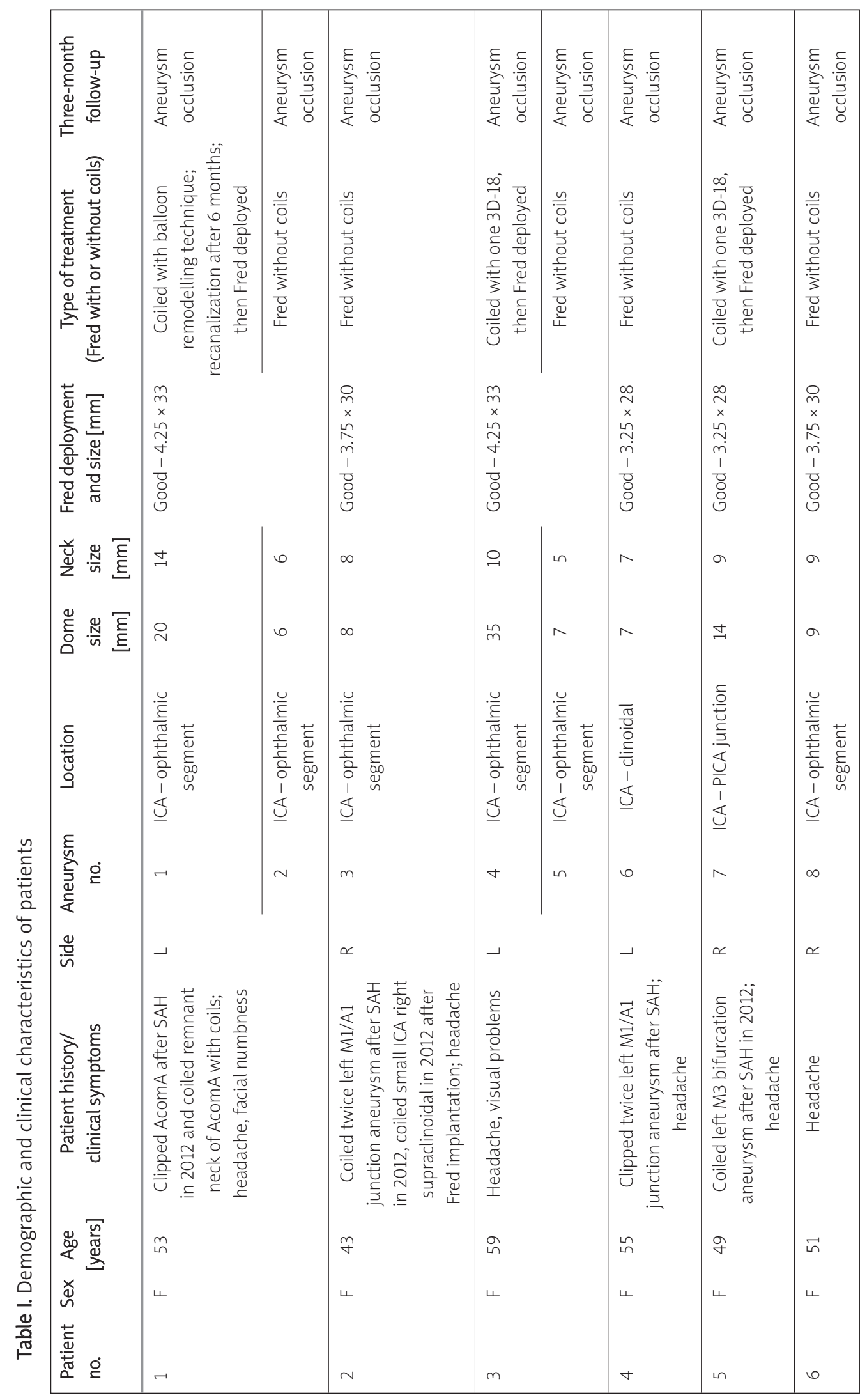



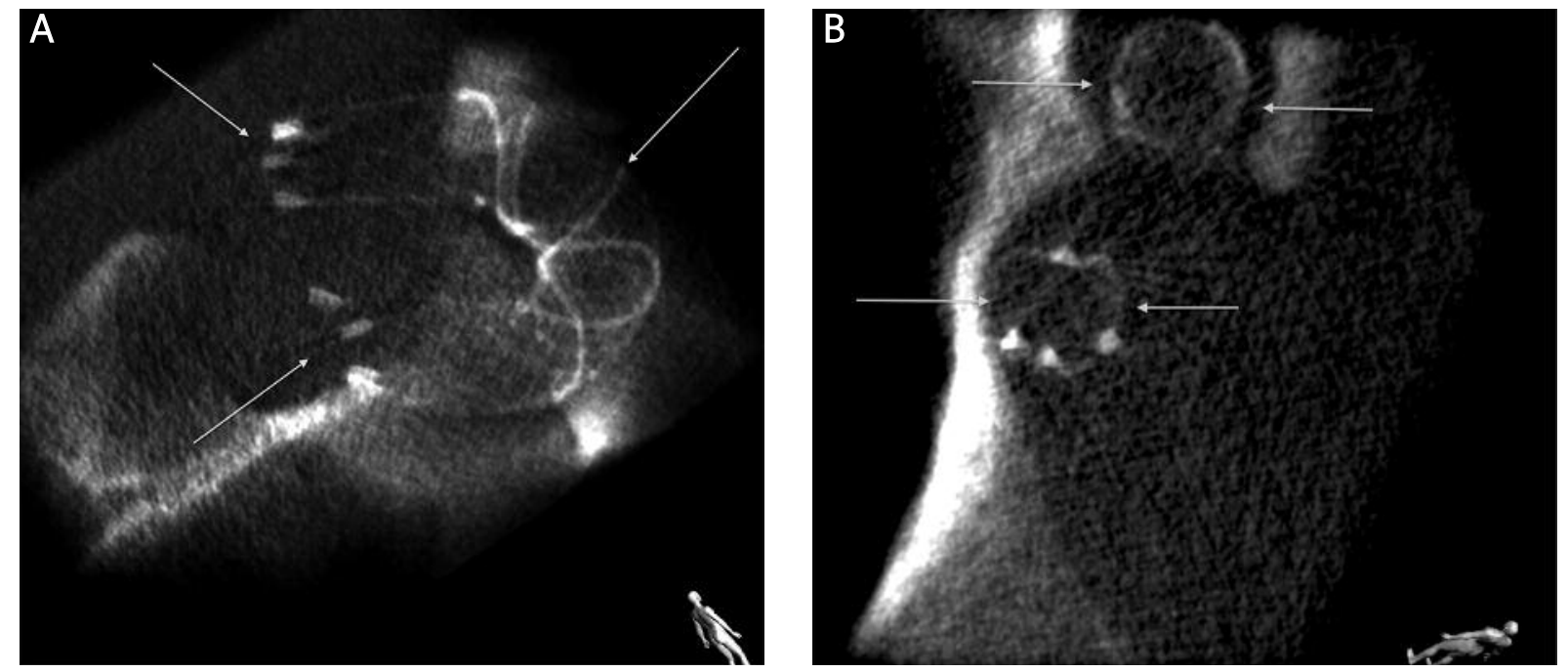

Photo 2. Case 2. A - Xpert-CT sagittal reconstruction shows flow diverter structure, position of proximal and distal markers as well as radio-opaque helix which demonstrates the working part of the device in relation to vessel wall (arrows). B - Xpert-CT coronal reconstruction shows full opening of the device (arrows)

3 months after was defined as no additional neurological changes in comparison to the status before the procedure using the modified Rankin score.

The haemodynamic success after 3 months was analyzed by a neurointerventional radiologist and neurosurgeon and was based on DSA defined as no flow inside the aneurysm sack and neck.

All 8 aneurysms in 6 patients were successfully stented with the Fred flow diverter device. All the patients were discharged in generally good condition. Initial technical success of the treatment was seen in all cases (Photos 3 A-D). The DSA control after 3 months showed complete occlusion of the aneurysms in 5 cases, and thrombosis of the Fred in one case (Photo $4 \mathrm{~A}$ ), where the constriction of the distal flared ends was present (Photo 4 B) without any other abnormalities which could explain the cause. The thrombosis occurred without clinical consequences because of flow from the other side (Photo $4 \mathrm{C}$ ). There was excellent clinical success before discharge from hospital and 3 months in all cases. No changes based on the modified Rankin score scale were observed. Only 1 patient reported headache after 3 months that disappeared after 5 months. One patient reported limited field of view and ptosis which persisted beyond. Haemodynamic success after 3 months occurred in all cases.

In 1 case haematoma in the groin was present without any surgical intervention. There were no adverse events that led to death or life-threatening injury connected with the equipment used or the procedure, or that caused permanent body impairment or required hospitalization with medical or surgical intervention as a result of any given complication.

\section{Discussion}

Wide-necked, complex or dissecting aneurysms are difficult to treat and require stents or new devices such as flow diverters to reconstruct the particular parent artery [1-17]. Different types of stents are currently available to use with the coils to support the neck of the aneurysms but some are poorly visualized, and some allow the coil to protrude into the parent artery because of the not fully opposed structure of the stent to the side of the artery or too large mesh spaces in the stent [17-22]. In some situations coil placement is not possible even with the use of stents. New devices such as flow diverters can help to solve such a situation and offer a different approach in treatment of those types of aneurysms. Usage of flow diverters is basically limited to unruptured aneurysms but some endovascular centres used them in ruptured cases as well [1-16].

The MicroVention (FRED first generation) flowdiverter system is a self-expanding nickel titanium, single wire braid, and compliant closed-cell design compatible with a 0.027-inch Headaway microcatheter (MicroVention). This compliant closed-cell device is designed to allow simultaneous deploy- 

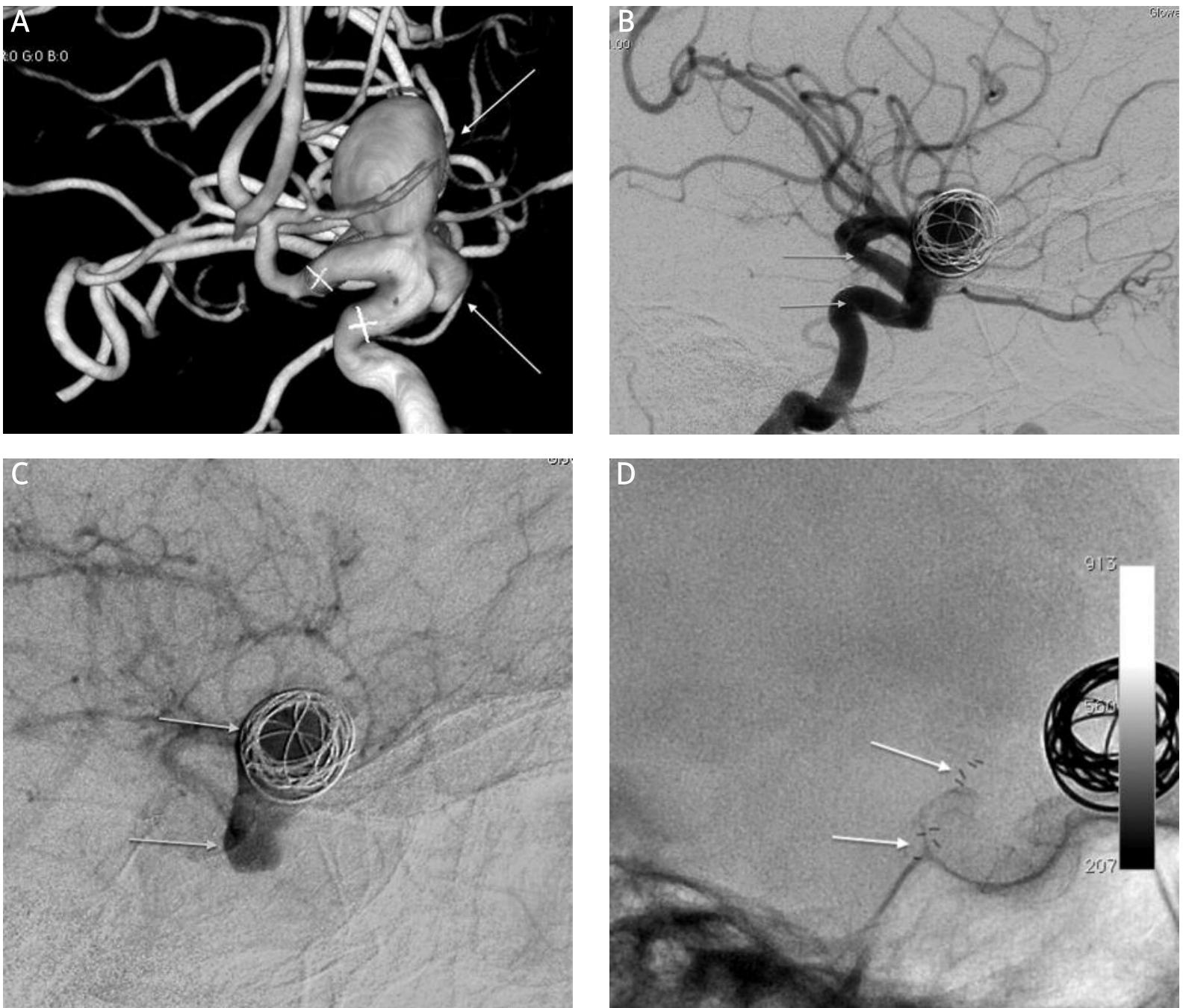

Photo 3. A - Oblique 3D reconstruction of arterial phase angiogram showing two combined internal carotid artery aneurysms in the ophthalmic segment (arrows) and flow diverter planning projection (mark X). B Lateral projection of arterial phase angiogram obtained just after 3D coil detechement and flow diverter deployment (arrows). C - Lateral projection of late angiogram phase-contrast stagnation in the aneurysms. D - Lateral projection native views flow-diverter proximal and distal markers show fully open flow-diverter system (arrows)

ment and retrieval by a single operator. The Fred system has dual working mid-section layer coverage marked with radio-opaque helix to provide fluoroscopic visibility, distal, and proximal markers on its single layer ends. This dual layer coverage is designed to limit the working layer mainly at the neck of the aneurysm and to create a flow diversion effect. Fred implantation relies on push and pull technique but the whole system can be re-constrained up to $80 \%$ of its deployment. The proximal and distal flared ends splay apart, showing that the proximal and distal ends of the device are open. The helical radio-opaque strands also spread into a double helix configuration, with alternating wall opposition on fluoroscopy. In all cases we performed XpertCT and Vaso-CT with a 10\% contrast diluted with saline which offered CT-like imaging to visualize bone, soft tissue, vessel morphology and flow diverter structure with good reconstruction which allow one to assess the position of the device in relation to the vessel wall. Metal artefact reduction is an improved algorithm on Vaso-CT that allows one to minimize the artefacts caused by the metal wire structure in the flow diverter. 
In our series Fred demonstrated $100 \%$ aneurysm occlusion. We had one case of ICA thrombosis, but cross-flow from the other side prevented clinical consequences. In this case constriction of the distal flared ends occurred, but it is not clear whether this phenomenon might play a role in the thrombosis. No deaths occurred during 3-month follow-up.

The current study presents a small case series of patients with a short observation period. It is impossible to compare the results reliably with previous large series. We know from a meta-analysis concerning flow-diverter implantations based on 29 studies and 1451 patients with 1654 aneurysms that morbidity and mortality rates were $5 \%$ and $4 \%$, respectively, and safety of flow diversion in small aneurysms was superior to that of large aneurysms [16]. The rate of post-procedural SAH or intraparenchymal haemorrhage was 3\% and 3\% [16]. The perforator infarction rate was $3 \%$ and ischaemic stroke rate was $6 \%$ with significantly lower infarction among patients with anterior circulation [16].

In a systematic review of the literature based on 97 studies with 7172 patients with intracranial unruptured aneurysms which were treated by endovascular technique other than flow diverter $1.8 \%$ died but unfavourable outcomes were reported in 4.7\% [17]. Significantly higher risk was associated with liquid embolic agents (8.1\%) compared to coil embolizations (4.9\%) [17]. Comparing the data from these two studies, we can assume that the risk of the use of a flow diverter is similar to the traditional method [16, 17]. Potentially favourable results of Fred in our case series could be associated with different flow diverter construction or the use of coils in association with flow diverters in cases of big aneurysms. We also had anterior circulation aneurysms which could have an impact on the lower complication rate compared to patients with posterior circulation [16]. It was reported and advised by Balt Extrusion not to use the Silk flow diverter without coils because of potential rupture of the aneurysm [7]. Our data cannot be used to compare the efficacy of the Fred flow-diverter device with other flow diverters or different endovascular technology used for aneurysm treatment because of the too small group of patients and short follow-up time.

\section{Conclusions}

Use of the Fred flow-diverter device was efficacious in all 8 treated cerebral aneurysms. The system
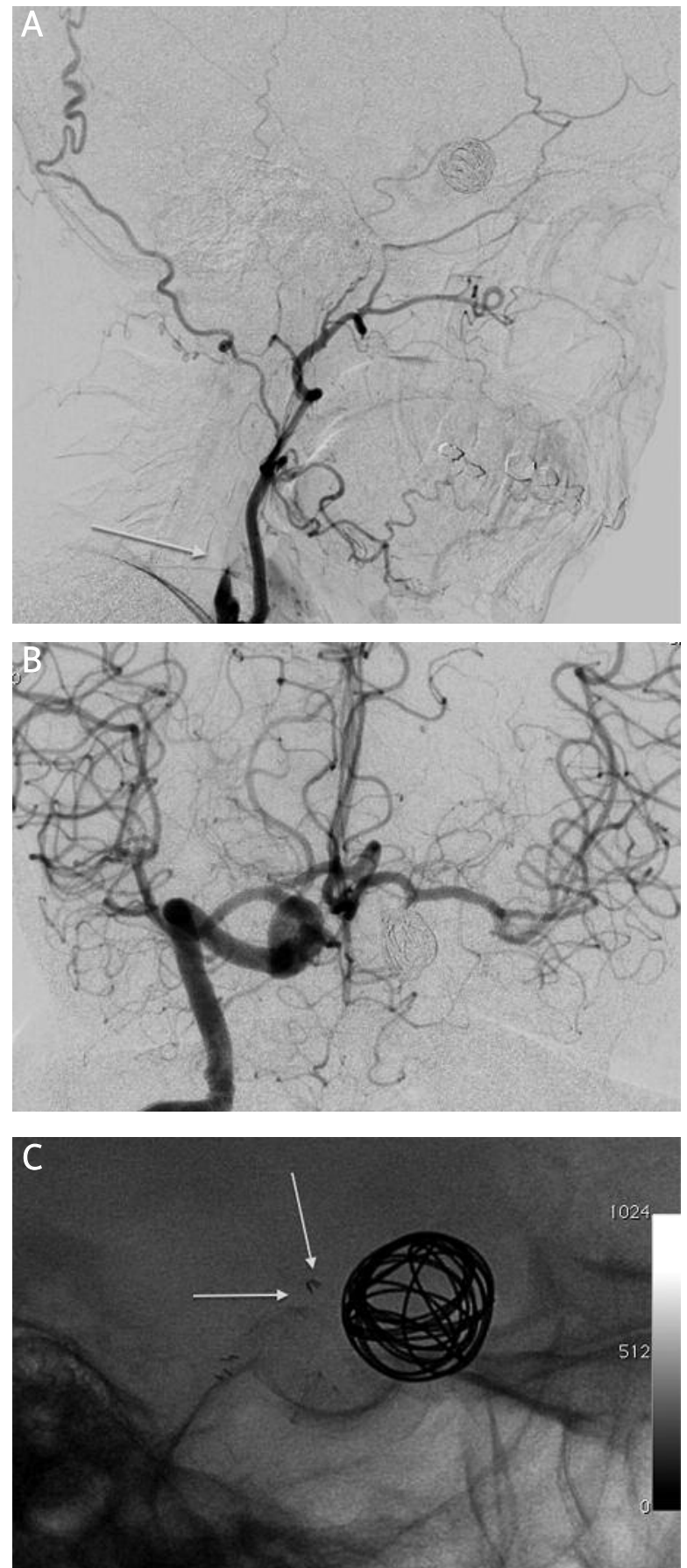

Photo 4. Case 3. A - Lateral projection of arterial phase angiogram obtained on 3-month followup shows occlusion of the left internal carotid artery aneurysm (arrows). B - Anterior-posterior projection of arterial phase angiogram obtained on 3-month follow-up shows cross-flow from right internal carotid artery aneurysm. C - Lateral projection-native views show pushed to the center distal markers of the flow diverter (arrows) 
seems to be promising as a flow diverter with certain characteristics, which allow for easy delivery and implantation. Further clinical evaluation with a larger group of patients is needed.

\section{Acknowledgments}

Supported by Grant PUM-11/12 Pomeranian Medical University Szczecin, Rybacka 1, Poland.

\section{References}

1. D’Urso PI, Lanzino G, Cloft HJ, Kallmes DF. Flow diversion for intracranial aneurysms: a review. Stroke 2011; 42: 2363-8.

2. Berge J, Biondi A, Machi P, et al. Flow-diverter silk stent for the treatment of intracranial aneurysms: 1-year follow-up in a multicenter study. AJNR Am J Neuroradiol 2012; 33: 1150-5.

3. Briganti F, Napoli M, Tortora F, et al. Italian multicenter experience with flow-diverter devices for intracranial unruptured aneurysm treatment with periprocedural complications - a retrospective data analysis. Neuroradiology 2012; 54: 1145-52.

4. Byrne JV, Beltechi R, Yarnold JA, et al. Early experience in the treatment of intra-cranial aneurysms by endovascular flow diversion: a multicentre prospective study. PLOS ONE 2010; 5: e12492.

5. Cruz JP, Chow M, O'Kelly C, et al. Delayed ipsilateral parenchymal hemorrhage following flow diversion for the treatment of anterior circulation aneurysms. AJNR Am J Neuroradiol 2012; 33: 603-8.

6. Deutschmann HA, Wehrschuetz M, Augustin M, et al. Long-term follow-up after treatment of intracranial aneurysms with the Pipeline embolization device: results from a single center. AJNR Am J Neuroradiol 2012; 33: 481-6.

7. Lubicz B, Collignon L, Raphaeli G, et al. Flow-diverter stent for the endovascular treatment of intracranial aneurysms: a prospective study in 29 patients with 34 aneurysms. Stroke 2010; 41: 2247-53.

8. Lylyk P, Miranda C, Ceratto R, et al. Curative endovascular reconstruction of cerebral aneurysms with the pipeline embolization device: the Buenos Aires experience. Neurosurgery 2009; 64: 632-42.

9. McAuliffe W, Wycoco V, Rice H, et al. Immediate and midterm results following treatment of unruptured intracranial aneurysms with the pipeline embolization device. AJNR Am J Neuroradiol 2012; 33: 164-70.

10. Nelson PK, Lylyk P, Szikora I, et al. The pipeline embolization device for the intracranial treatment of aneurysms trial. AJNR Am J Neuroradiol 2011; 32: 34-40.

11. Saatci I, Yavuz K, Ozer C, et al. Treatment of intracranial aneurysms using the pipeline flow-diverter embolization device: a single-center experience with long-term follow-up results. AJNR Am J Neuroradiol 2012; 33: 1436-46.

12. Szikora I, Berentei Z, Kulcsar Z, et al. Treatment of intracranial aneurysms by functional reconstruction of the parent artery: the Budapest experience with the pipeline embolization device. AJNR Am I Neuroradiol 2010; 31: 1139-47.
13. Velioglu M, Kizilkilic O, Selcuk H, et al. Early and midterm results of complex cerebral aneurysms treated with Silk stent. Neuroradiology 2012; 54: 1355-65.

14. Wagner A, Cortsen M, Hauerberg J, et al. Treatment of intracranial aneurysms. Reconstruction of the parent artery with flowdiverting (Silk) stent. Neuroradiology 2012; 54: 709-18.

15. Yu SC, Kwok CK, Cheng PW, et al. Intracranial aneurysms: midterm outcome of pipeline embolization device - a prospective study in 143 patients with 178 aneurysms. Radiology 2012; 265: 893-901.

16. Brinjikji W, Murad MH, Lanzino G, et al. Endovascular treatment of intracranial aneurysms with flow diverters a meta-analysis. Stroke 2013; 44: 442-7.

17. Naggara ON, Lecler A, Oppenheim C, et al. Endovascular treatment of intracranial unruptured aneurysms: a systematic review of the literature on safety with emphasis on subgroup analyses. Radiology 2012; 263: 828-35.

18. Lubicz B, François O, Levivier M, et al. Preliminary experience with the enterprise stent for endovascular treatment of complex intracranial aneurysms: potential advantages and limiting characteristics. Neurosurgery 2008; 62: 1063-9.

19. Mocco J, Snyder KV, Albuquerque FC, et al. Treatment of intracranial aneurysms with the Enterprise stent: a multicenter registry. J Neurosurg 2009; 110: 35-9.

20. Krischek O, Miloslavski E, Fischer S, et al. A comparison of functional and physical properties of self-expanding intracranial stents [Neuroform3, Wingspan, Solitaire, Leo+, Enterprise]. Minim Invasive Neurosurg 2011; 54: 21-8.

21. Biondi, Janardhan V, Katz JM, et al. Neuroform stent-assisted coil embolization of wide-necked intracranial aneurysms: strategies in stent deployment and midterm follow-up. Neurosurgery 2007; 61: 460-8.

22. Benitez, Silva MT, Klem J, et al. Endovascular occlusion of widenecked aneurysm with a new intracranial microstent (Neuroform) and detachable coil. Neurosurgery 2004; 54: 1359-67.

Received: 20.04.2013, accepted: 17.05.2013. 